\title{
How Clinicians Discuss Medications During Primary Care Encounters Among Older Adults with Cognitive Impairment
}

\author{
Ariel R. Green, $M D, M P H, P h D^{7}$, Jennifer L. Wolff, $P h D^{2}$, Diane M. Echavarria, $M S^{2}$, \\ Malcolm Chapman, $B A^{3}$, Annie Phung, $\mathrm{MS}^{4}$, Devon Smith, BA ${ }^{5}$, and Cynthia M. Boyd, \\ $\mathrm{MD}, \mathrm{MPH}^{\top}$
}

'Division of Geriatric Medicine and Gerontology, Department of Medicine, Johns Hopkins University School of Medicine, Baltimore, MD, USA; ${ }^{2}$ Roger C. Lipitz Center for Integrated Health Care, Department of Health Policy and Management, Johns Hopkins Bloomberg School of Public Health, Baltimore, MD, USA; ${ }^{3}$ University of North Carolina School of Medicine, Chapel Hill, NC, USA; ${ }^{4}$ Philadelphia College of Osteopathic MedicineGeorgia, Suwanee, GA, USA; ${ }^{5}$ Northeast Ohio Medical University, Rootstown, OH, USA.

BACKGROUND: People with cognitive impairment experience high rates of polypharmacy and potentially inappropriate medication use. How clinicians communicate about medications may affect to what extent patients and family companions understand and participate in decisions about medication use.

OBJECTIVE: To characterize how primary care clinicians discuss medications during encounters with older adults with cognitive impairment and their companions.

DESIGN: Qualitative content analysis of audio-recorded clinical encounters from SAME Page, a randomized controlled trial to examine the effects of a patient-family agenda setting checklist on primary care visit communication among patients with cognitive impairment. Visits occurred between August 2016 and August 2017.

PARTICIPANTS: Patients were 65 or older, had $>1$ incorrect answer on a cognitive screener, and attended visits with a relative or unpaid companion. Clinicians were physicians, nurse practitioners, or physician assistants at participating practices.

APPROACH: The encounters were transcribed verbatim. We used qualitative content analysis to identify major themes.

KEY RESULTS: Patients were on average 79.9 years of age. The average MMSE score was 21.6. About half of clinicians reported practicing for 15 or more years $(n=8)$. We identified three major themes. First, we found numerous instances in which primary care clinicians introduced patients and companions to key principles of optimal prescribing and deprescribing. Second, clinicians used a variety of approaches to foster shared decision-making about medication use. Third, several challenges prevented clinicians from working together with patients and companions to optimize prescribing and deprescribing.

CONCLUSIONS: This study offers insight into key language clinicians can use to initiate discussions about optimizing prescribing, as well as barriers they face in doing so. Examples identified in these transcripts should be tested with patients and caregivers to examine how such communications are received and interpreted.

Prior presentations: None.

Received January 25, 2019

Revised July 19, 2019

Accepted September 6, 2019

Published online November 8, 2019
Future research should develop and test interventions that seek to overcome obstacles to optimizing prescribing for older adults with cognitive impairment.

KEY WORDS: shared decision-making; primary care; dementia.

J Gen Intern Med 35(1):237-46

DOI: $10.1007 / \mathrm{s} 11606-019-05424-6$

() Society of General Internal Medicine 2019

\section{INTRODUCTION}

An estimated 11 million Americans are living with cognitive impairment, ${ }^{1,2}$ a condition associated with increased disability, neuropsychiatric symptoms and health care costs, and decreased quality of life. ${ }^{3,4}$ People with cognitive impairment have a high burden of coexisting medical conditions, making them likely to experience polypharmacy, potentially inappropriate medication (PIM) use, medication side effects, adverse drug events, and treatment burden. ${ }^{1,5-10}$ For example, people with dementia take 5-10 medications, on average, of which 12 are prescribed for dementia and the remainder for coexisting, non-dementia illnesses. ${ }^{6}$ One-third of people with dementia take PIMs, in which the harms of medications may outweigh the benefits. ${ }^{6}$

For clinicians, achieving optimal medication use for older adults with cognitive impairment is challenging. Decisions about medication use in such patients often carry trade-offs between quality and length of life, or between different facets of quality of life. For example, many medications commonly used to treat incontinence, insomnia, and pain have anticholinergic properties ${ }^{11}$, which can cause dry mouth, constipation, falls, and delirium. ${ }^{12-16}$ Anticholinergic medications may also be associated with long-term, permanent cognitive decline. ${ }^{17}$ Given the prolonged and variable course of cognitive impairment, determining whether a medication to prevent or treat a coexisting condition is likely to result in benefits or harms during the person's remaining lifespan is complex. Medications that were once appropriate may become inappropriate as cognition declines; exactly when this shift occurs may be difficult to determine. Lack of clinical trial data, guidelines, 
or decision support tools applicable to patients with cognitive impairment leaves clinicians forced to make prescribing decisions without much guidance. ${ }^{18}$

Optimizing medication use for people with cognitive impairment often involves deprescribing - tapering or stopping drugs to reduce inappropriate polypharmacy. Two recent reviews of deprescribing emphasized the importance of shared decisionmaking with patients and family caregivers. ${ }^{19,}{ }^{20}$ When patients and caregivers engage in shared decision-making, they become better informed about risks and benefits and tend to choose fewer medicines..$^{21}$ However, one barrier to optimizing prescribing may be clinician discomfort in discussing topics such as life expectancy and in elicitation of patient and caregiver preferences. ${ }^{18}$ This barrier may be heightened in people with cognitive impairment due to communication difficulties and weakened decision-making capacity. ${ }^{22}$ Furthermore, people with cognitive impairment are often accompanied to medical visits by family members or other companions, meaning that clinicians must be skilled at engaging and weighing multiple perspectives during the encounter. ${ }^{23}$

How clinicians communicate about medications during the clinical encounter may affect to what extent patients and family companions understand and participate in decisionmaking about medication use. This is particularly important for people with cognitive impairment because they face more complex decisions and greater risks from medications. Little is known about how clinicians can best facilitate shared decision-making about optimal medication use among patients with cognitive impairment and their companions. To begin developing a framework for fostering shared decision-making about medication use for people with cognitive impairment, the objective of this study was to characterize how primary care clinicians currently discuss medications during encounters with older adults with cognitive impairment and their family companions. We used qualitative methods to analyze medication discussions from audio recordings of patients' primary care visits from the SAME Page Study, a randomized controlled trial to examine the effects of a patient-family agenda setting checklist on primary care visit communication among older adults with cognitive impairment. ${ }^{24}$

\section{METHODS}

\section{Overall Study Design}

We performed a qualitative content analysis of audio-recorded clinical encounters from the SAME Page trial. The trial did not intervene upon clinicians and did not address optimal prescribing and deprescribing. Further details are published elsewhere. ${ }^{24}$

\section{Study Participants}

SAME Page enrolled 93 patient-companion dyads at 2 primary care clinics and 1 hospital-based geriatrics clinic in Baltimore, Maryland. Eligible patients were 65 or older,
English speaking, had at least 1 incorrect answer on a cognitive screener, ${ }^{25}$ and attended primary care visits with a relative or unpaid companion. Eligible clinicians were practicing physicians, nurse practitioners, or physician assistants at the 3 participating primary care practices. Fourteen clinicians met eligibility criteria and agreed to participate. The study was approved by the institutional review board of the Johns Hopkins Bloomberg School of Public Health.

\section{Data Collection and Analysis}

Patient diagnoses and medications were abstracted from the electronic medical record. The Mini-Mental State Examination (MMSE) was administered by research staff at the enrollment visit. Clinician characteristics were assessed from surveys administered at the time of informed consent. Dyads in the intervention arm completed a self-administered checklist in the waiting room to establish a shared visit agenda. Dyads in the control group waited for their visit as usual. Patients and companions also completed a 2-week follow-up questionnaire, which included questions about medication adherence and communication with clinicians, both in general and specifically relating to prescription medications. Response categories for the communication question were poor, fair, good, and excellent. Visits were audio-recorded and transcribed verbatim. All 93 recordings were included in the qualitative analysis.

We developed a preliminary coding scheme after an initial reading of 6 transcripts by a multidisciplinary team (A.R.G., D.E., C.M.B., and J.L.W.) with expertise in geriatric medicine, deprescribing, family caregiving, dementia, and qualitative research. Four investigators (A.R.G., M.C., D.S., and A.P.) refined the coding scheme by reading 12 additional transcripts. Coding was subsequently performed by these 4 team members using Atlas.ti, version 8 (ATLAS.ti Scientific Software Development). Each transcript was independently read by 3 coders. The constant comparative approach was used to qualitatively analyze the content of the transcripts. ${ }^{26,27}$ Open coding allowed inductive identification of new themes in addition to deductive coding ${ }^{28}$ guided by shared decision-making frameworks. ${ }^{29,}{ }^{30}$ At weekly meetings, the transcripts were reviewed and assessed for the emergence of new ideas or themes. Differences were reconciled by consensus until $100 \%$ agreement was reached. Revisions to the coding scheme were applied to all previously coded transcripts. Content analysis generated major themes and subthemes. Visits occurred between August 2016 and August 2017. Data analysis was performed between June 4 and July 27, 2018.

\section{RESULTS}

\section{Clinician Characteristics}

Clinicians were on average 49.3 years of age (SD, 11.1; range, 29-62). About half reported practicing 15 or more years $(n=$ 8 ; Table 1). Clinicians were trained in family practice $(n=6)$, 
Table 1 Characteristics of Primary Care Clinics, Clinicians, and Study Participants

\begin{tabular}{ll}
\hline \hline Characteristic & No. $(\%)$ \\
\hline Patients $(N=93)$ & \\
Age, mean (SD) (years) & $79.9(7.6)$ \\
Female gender & $48(51.6)$ \\
Nonwhite, Hispanic, or other & $39(41.9)$ \\
Beyond high school education & $45(48.4)$ \\
Mini-Mental State Examination score, mean (SD) & $21.6(6.8)$ \\
10 prescription medications & $22(23.7)$ \\
Companions $(N=93)$ & \\
Female gender & $70(75.3)$ \\
Relationship to patient & \\
Spouse/partner & $37(39.8)$ \\
Adult child & $51(54.8)$ \\
Other (friend, other relative) & $5(5.4)$ \\
Beyond high school education & $67(72.0)$ \\
Clinicians $(n=14)$ & \\
Female gender & $7(50.0)$ \\
Type of training & \\
Family medicine & $6(42.8)$ \\
Internal medicine & $3(21.4)$ \\
Nurse practitioner or physician assistant & $5(35.7)$ \\
Specialty training in geriatrics & $6(42.9)$ \\
Years in practice & \\
<5 years & $2(14.3)$ \\
5-15 years & $4(28.6)$ \\
>15 years & $8(57.1)$ \\
Proportion of panel aged 65+ & \\
<25\% & $2(14.3)$ \\
25-49\% & $535.7)$ \\
$50 \%+$ & $7(50.0)$ \\
\hline
\end{tabular}

internal medicine $(n=3)$, or were nurse practitioners or physician assistants $(n=5)$. Most ( $n=5$ of 6 ) clinicians with geriatrics training practiced at the geriatrics clinic.

\section{Patient and Companion Characteristics}

Patients were on average 79.9 years of age (7.6). About half of enrolled patients $(49.5 \%)$ had a diagnosis of dementia, cognitive impairment, or symptoms of cognitive impairment recorded in their electronic health record. We will hereafter refer to this population as having cognitive impairment, a category which ranges from mild to severe. Patients' average MMSE score was 21.6 (6.8). Most companions were female (75.3\%) and were spouses $(39.8 \%)$ or adult children $(55.8 \%)$ of patients; few $(5.4 \%)$ were friends or other relatives.

\section{Nature of the Encounters}

The visits lasted a mean of $25.2 \mathrm{~min}$ (SD, 13.8; range, 6.01$57.0)$. The majority of patients (52\%) and companions (63\%) who responded to the question about quality of medication communication with the clinician rated it as excellent (Appendix Table 4). Our qualitative content analysis clustered into three major categories, or themes, and associated subcategories. Below, we describe each theme; selected representative quotes are presented in the tables.

Theme 1: Key principles of optimal prescribing discussed by clinicians. We found numerous examples of clinicians introducing patients and companions to key principles of optimal prescribing for older adults with cognitive impairment. These principles are presented in Table 2, along with representative quotes. Clinicians talked of scaling back the intensity of certain medications and other interventions as a routine part of care in order to avoid treatment burden and improve quality of life, as in this encounter with an 82-yearold patient with a MMSE score of 26 who routinely saw four different physicians and took multiple medications that required monitoring (digoxin, warfarin, and levothyroxine); the patient's health status was further complicated by "sundowning":

Clinician: One of the things that I like to do is to try and simplify a person's life and I do that by simplifying medications where I can and... also... by trying to simplify doctors that you see.

Clinicians further laid the groundwork for conversations about deprescribing by explaining that a person's needs, and the balance of benefits and harms of medications, change with aging and cognitive impairment. They often prioritized function and quality of life over the traditional, disease-based approach to care, particularly for older adults with more severe chronic conditions or poorer cognitive status. They explained the concept of diminishing returns - i.e., when the risk of a negative health outcome, such as a cardiovascular event, can be reduced in several different ways, the benefit of each successive intervention is modest and further attenuated by iatrogenic harm. Clinicians helped patients and companions understand that medications usually do not "fix" every symptom and that they carry risk. They also recognized the uncertainty of the medical evidence applicable to older adults with dementia and coexisting conditions, thereby acknowledging the preference-sensitive nature of medication decisions.

Theme 2: Clinician strategies to establish partnership with patients and companions. Clinicians used a variety of approaches to develop partnerships with patients and companions and to foster shared decision-making about deprescribing: eliciting the patient or companion perspective; providing reassurance that deprescribing is not a withdrawal of appropriate care; and providing reassurance about ongoing care and monitoring during the deprescribing process. For example, in this encounter with a 78-year-old man (MMSE 26), the clinician first made the patient aware that his anticholinergic medication could be contributing to his recurrent falls, and then sought the patient's perspective on stopping the medication:

Now we have an event that is happening [i.e., falls], we need to modify. Down the road, if I want to take you off of the amitriptyline and replace it with something for neuropathy, would you be okay with that? 
Table 2 Key Principles of Optimal Prescribing Discussed by Clinicians

\begin{tabular}{lcl}
\hline \hline Principle & Example & Perceived quality of \\
communication with clinician & about medications* & \\
about & &
\end{tabular}

Balance of benefits and harms may change with aging and cognitive impairment

Prioritizing function and quality of life over disease-based approach to care

Diminishing returns ${ }^{\dagger}$

Symptoms cannot always be "fixed" with medication

Uncertainty of evidence for older adults with cognitive impairment and competing health risks
Patient has had multiple hypoglycemic episodes.

Clinician: Normally in diabetes, we think about the risk of kidney disease, the risk of eye disease... but as we get older, the risk of a low sugar reaction is actually more important... You could certainly go into a situation where... you fall, you hit your head and break your hip.

- 75 years old, MMSE 25 , usual care, general primary care clinic Patient has visual and hearing impairment, depression and difficulty managing medications.

Clinician: What else would make you stronger and more healthy? What would you like to do yourself or like us to do?...What would make you feel better and more independent and safe...?

Patient: ...What I would like to start doing is driving again, but I mean...I'm not ready for it right now.

- 82 yo, MMSE 28, intervention, geriatrics clinic

Patient has recurrent falls.

Clinician: What I might suggest is the fact that [your blood pressure] doesn't have to be as tightly controlled as it is today. [Explains that the patient is taking three antihypertensive medications.]...I think we need to...reduce the amount of medicine that you're taking, because... they can interfere with falling.

- 78 yo, MMSE 26, intervention, geriatrics clinic

Patient says he plans to ask urologist about increasing

tamsulosin dose because he is getting up too often at night to urinate.

Clinician: It's a fair enough goal, but it's tough...There's always simple things like...eliminating your fluid intake in the evening hours.

Patient: Oh, it's like the discussions we've had before about you're growing older and there are things happening to your body...And your answer was, "Well, does it affect your quality of life?...If it doesn't, then maybe it's just something you just... deal with..."

Clinician: There's no easy answer, I guess, but part of the flip side is that...in medicine, we want to do no harm.

- 70 yo, MMSE 29, intervention, general primary care clinic

Patient is taking aspirin and rivaroxaban and has "balance issues"

Clinician: The problem with blood thinners is that if you fall and injure yourself, you can bleed. But it's a risk versus a benefit... There are people who are three and four of these medicines... Now they're at great risk to have bleeding, but they're also at great risk to have blood clots... The reason why I have a lot of gray hair, is that it's a constant battle to...take people off medicine and put people on medicine.

- 76 years old, MMSE 20, intervention group, general primary care clinic
Patient: High

Companion: High

Patient: Missing

Companion: Low

Patient: Low

Companion: High

Patient: High

Companion: High

Patient: High

Companion: High

yo years old

*Asked as part of a 2-week follow-up questionnaire: low = poor, fair, good; high = excellent

†Diminishing returns: When the risk of a negative health outcome, such as a cardiovascular event, can be reduced in several different ways, the benefit of each successive intervention is modest and further attenuated by iatrogenic risks 31

The clinician also emphasized that he did not intend to withdraw appropriate care:

I want to get to know you a little bit more...And then work together on minimizing your medications. Understanding that I need to take care of your neuropathy, I need to make sure you're sleeping okay, and I need to make sure your blood pressure is okay. So I will not sacrifice what needs to be done, but I will try to minimize the amount of medicine that we use.
Phrases such as "Would you be okay with that" and "I want to get to know you" foster elicitation of the patient's priorities. Clinicians also reassured patients and companions that they would monitor closely for returning symptoms after stopping a medication, as in this encounter with an 81-year-old patient (MMSE 26): "I think you're going to do fine without [amlodipine]. We'll check you again down the...short road."

Theme 3: Factors complicating optimal prescribing. Clinicians had to balance the principles of optimal prescribing against other considerations. These 
considerations are presented in Table 3. Factors complicating optimal prescribing included the following: distressing behavioral and psychological symptoms of dementia; patient or companion expectations of receiving a prescription; discordance between clinician recommendations and patient or companion preferences; the challenges of triadic communication, particularly involving people with cognitive impairment; and the complexity of the patient's health status, missing records, or technical or logistical issues-which sometimes left little time for discussions about optimal prescribing or deprescribing, even when patients or companions explicitly mentioned concerns about medication appropriateness or treatment burden. For example, medication reconciliation took up a large portion of some visits, particularly when the patient was accompanied to the visit by a companion who was not a primary caregiver.

Clinicians struggled with balancing disease-based guidelines against quality of life, treatment burden, and side effects, as in this interaction between a clinician and the companion of an 83-year-old woman with a MMSE score of 12:

Clinician: The lisinopril and the metoprolol both are good for her heart and make it pump better. So I'd rather have that and put up with a little bit of a problem than have her going into congestive heart failure. But if [her dizziness] gets worse we can always take that lisinopril [and] drop it down to 2.5 [mg].

Clinicians had to navigate between patients and companions in their communication, which further complicated attempts to discuss optimal prescribing. With patients who had more advanced cognitive impairment and spoke little, clinicians tried to address them directly, to the extent possible, and determine what mattered most to the patient, as in the following interaction with a 74-year-old patient with a MMSE score of 12:

Companion: A lot of times... she sits there and cries... in pain and doesn't ask for it... And I have to kind of ask her sometimes and then I get frustrated...

Clinician: [Addressing patient] Would it make it better if we just make it that you get [acetaminophen]... a couple of times a day so you don't even have to ask?... I hate to add it on to the other drugs... Miss [Patient], is there anything that you can think of that I can do to help to make your life better?

Clinicians were particularly challenged to engage patients who had poor insight due to cognitive impairment, or when there was discord between patients and companions, as in the following interaction with a 66-year-old patient with an MMSE score of 13 :
Companion: It's still like kind of sporadic where there's confusion with pictures as actual people being in the house.

Patient: ...I beg to differ...

Clinician: [Addressing companion] How many times do you notice, like, a week?

Companion: Maybe once or twice a day...

Patient: [Addressing companion] It really infuriates me when you don't listen to me and you jump to so many conclusions... I'm not crazy. I'm not stupid...

Clinician: [Addressing patient] He just wants to let us know just so we can make sure that you're not getting any more infections or nothing else is happening.

In such interactions, clinicians often had to obtain history from the companion and then assure the patient that they were not being undermined to avoid upsetting them.

\section{DISCUSSION}

In this qualitative, observational study, we characterized primary care clinicians' approaches to discussing medication use during encounters with cognitively impaired older adults and their family companions. Despite high rates of polypharmacy and PIM use, few investigations have detailed the elements of communication about medication use in clinical care. To our knowledge, this is the first study to use audio-recordings of actual conversations between clinicians and older adults with cognitive impairment and their companions to describe the current state of communication about medication use.

Deprescribing protocols have been developed ${ }^{20,32,33}$; however, a barrier to their implementation is that clinicians find it challenging to elicit patient preferences and goals and to discuss concepts such as disease trajectory, estimated lifespan and ambiguity about benefits and harms - particularly given high levels of clinical complexity and time limitations. ${ }^{34,} 35$ We present examples in which clinicians introduced deprescribing to patients and companions during primary care encounters, with the goal of beginning to develop a framework for fostering shared decision-making about medication use for people with cognitive impairment. Clinicians discussed concepts such as the uncertainty of the evidence for many medications, particularly for people with cognitive impairment and other chronic conditions - a critical part of informed decision- 
Table 3 Factors Complicating Optimal Prescribing

\begin{tabular}{lcl}
\hline \hline Complicating factor & Example & Perceived quality of \\
communication with clinician & & \\
about medications* &
\end{tabular}

Distressing behavioral and psychological symptoms of dementia

Patient or caregiver expectations

Discordance between clinician recommendations and patient or caregiver preferences

Clinician attention diverted by complexity of patient's health status, missing records, and technical or logistical issues
Companion: [Discussing patient's recent ED visit for a fall.] I had given him the Risperdal. I don't think that caused it, but I don't know...

Clinician: ...Any of them can do it...We use them sparingly kind of off-label...to make sure that you're sleeping...You're $100 \%$ correct, the higher the dose, you tend to have an increased risk of falls, so the less we can get away with...the better...Sometimes the Namenda can cause a little bit of dizziness, so it could have been that. Companion: ...We did increase that at the last visit also. Clinician: ... That tends to kind of really help with agitation and just kind of calm things down a little bit. So the description you're giving me is kind of what we like the Namenda to do.

Companion: Yeah, it seems to be working well.

- 85 yo, MMSE 6, intervention, geriatrics clinic

Patient: I need something now with my bladder.

Clinician: [After eliciting more about the patient's symptoms] There's a couple issues with this, as far as treating versus not treating... The medicines that we use to slow the bladder down, kind of have side effects... The side effects can be things like dry mouth. But they can also be like dizziness... We have to be very, very care - but look, it's always a risk versus a benefit... I have a patient about your age, actually, who had a similar problem... She was not getting good sleep...because she was having to pee all the time...She was really miserable. And I mean, we were able to put her on a very small dose of a medicine. And she's like a new woman. I mean, I got to tell you, it's really incredible... [Writing prescription for oxybutynin] I wouldn't give it to you if I didn't think it wasn't safe. Because I just want to be clear that, you know, it can have these subtle effects on you.

- 83 yo, MMSE 24, intervention, general primary care clinic

Clinician: The biggest thing today is to get your medications straightened out and what we can trim up we're going to trim up...

Companion: [Describing the patient's recent hospital discharge.] So this young boy comes in, young doctor comes in...It was the cholesterol medicine, the atorvastatin. I said, 'Why does he have to discontinue that?' 'Oh, well studies show that after the age of 70 it's not very effective.' I said, 'Well I bet you we could find studies that show that it is...I said, 'All right, why does he have to stop the Nexium?' I said, 'He has acid reflux.' 'Oh, well maybe he could continue that.' And I said, 'I'm assuming you don't want him to have Celebrex because it causes strokes,' I said, '...He's got terrible arthritis in his back and his hips, his knees...I realize this causes strokes, but he's been taking it for ten years, I'm not stopping it.'... They've known him for three days... and all of a sudden they become experts on what they have to stop.

-100 yo, MMSE 9, intervention, geriatrics clinic Clinician: I suggested half a pill twice a day...This still says one tablet twice a day...Do you know offhand what you're taking? Or do you cut the pill in half?

Patient: I know I'm taking half a pill in the morning. Clinician: ....Did you see the heart doctor since I saw you last?

Patient: I don't think so.

Clinician: Because there's a note here - so we get some records through the computer but not complete...It said that on the 12th of last month that there was a visit with Dr. [Specialist] but there's no details of the visit...I want to see if Dr. [Specialist] is available real quick just to kind of review this with him because I'm a little nervous about your pressure. And it's really important to confirm exactly what dosages you're taking of the different medicines. So give me a second. I'll be right back...

[Clinician leaves room to call specialist in another health care system.]
Patient: Low

Companion: High

Patient: High

Companion: Low

Patient: Missing

Companion: High

Patient: High

Companion: High 


\begin{tabular}{|c|c|c|}
\hline Complicating factor & Example & $\begin{array}{l}\text { Perceived quality of } \\
\text { communication with clinician } \\
\text { about medications* }\end{array}$ \\
\hline
\end{tabular}

Triadic communication involving patients with cognitive impairment

Balancing disease-based guidelines with quality of life and treatment burden
Clinician: ...Dr. [Specialist] is actually out of town...The doctor who is covering there... had your records... Because of your heart problems he'd really like to kind of keep the blood pressure around 100 because it's easier for your heart...But if there's a concern about you getting lightheaded... and risk of falling then we would want the pressure a little higher...

Companion: He's taking what's on the list here. My mom gave me this to bring in.

Clinician: No pills are cut in half?

Companion: Whatever the list - well, he gets a half pill.

Patient: I get a half pill in the morning.

Clinician: But there's no pills saying half on the list. So that's what's critical. So it has to be a current and

completely accurate. What's the pill? What's the strength?

How many do you take? How often do you take it?

- 70 yo, MMSE 24, intervention group, general primary

care clinic

Clinician: What I would suggest is to go up on the Patient: Low

citalopram.

Patient: The what?

Clinician: Citalopram, that is the -

Companion: That's one that we talk about being for her

blood pressure. We don't talk about it.

Clinician: Mm-hmm. Okay..

Companion: And she's been actually also asking does she need the aspirin?

Patient: The aspirin? I never said anything about any aspirin... I don't like to be fed medicine constantly... Companion: Well, it's not constant mother. It's once in the morning and once at night...

Clinician: You know, we're only going to suggest things

that we think are necessary... A number of your blood

pressure medicines work to get your pressure down in different ways. And so that's why I'd like to go up on that one [referring to citalopram].

- 78 yo, MMSE 20, intervention, general primary care clinic

Patient: I take that, my Lipitor and, well I take 5 pills in the morning, 9 after I eat and 5 more in the evening, bedtime, 19 pills, that's...

Clinician: Let's check your blood pressure...

Clinician does not address patient's comment about taking 19 pills for remainder of visit.

Clinician: Your diabetes was perfect...In September you were at 5.8 so we don't need to do anything different, I'll just see you back in six months for your complete physical, okay?

-85 yo, MMSE 30, intervention, general primary care

clinic
Companion: High

Patient: Low

Companion: Low

yo years old

*Asked as part of a 2-week follow-up questionnaire: low = poor, fair, good; high = excellent

making. We also observed clinicians asking patients and companions what health problems and symptoms they wanted to prioritize - treating the person, rather than the disease - and making it clear that deprescribing recommendations were designed to improve quality of life, not because the patient was no longer "worth treating."19 If confirmed in different types of populations, our results suggest that it is possible for clinicians to facilitate conversations about deprescribing as part of the routine care of people with cognitive impairment.

Previous research has shown that patients and companions may not recognize the potential harms of medication use or be aware that deprescribing is possible. For example, a recent study found that most caregivers of people with dementia believed antipsychotic medications used to treat behavioral and psychological symptoms of dementia were safe, despite these drugs' well-documented potential for severe side effects. ${ }^{36}$ Other studies have found older adults and companions to be open to the idea of deprescribing if their clinician recommends it, ${ }^{37,}{ }^{38}$ but often feel that they are unable to obtain information about medications or make their preferences heard. ${ }^{39,}{ }^{40}$ By analyzing the content of conversations about medication use, our study offers additional insight into key language clinicians can use to initiate discussions about deprescribing, as well as barriers they face in doing so. For 
example, it would appear from these transcripts that clinicians often struggle to deprescribe preventive medications in people with cognitive impairment, in part because of the difficulty of extrapolating risk-benefit data to individual patients, and in part because prescribing is seen as a positive action, while deprescribing is not. ${ }^{19}$ Risk prediction tools available at the point of care would enable clinicians to calculate how much each recommended medication (e.g., ACE inhibitor and betablocker for a patient with systolic heart failure) contributes to absolute risk reduction and whether the benefit of using both medications justifies the potential harms for patients with cognitive impairment, who are at increased risk of adverse drug effects. ${ }^{31}$ Existing risk prediction calculators ${ }^{41}$ and decision aids ${ }^{21}$ generally do not incorporate cognitive impairment and may perform poorly for older adults with multiple competing risks of mortality. ${ }^{42}$ Future research is needed to generate evidence to inform the development of risk prediction calculators applicable to people with cognitive impairment.

Our data do not enable us to comment on encounters in which neither the clinician nor the patient or companion raised the issue of medication appropriateness or deprescribing. However, we identified missed opportunities to discuss optimal prescribing, even when patients or companions explicitly mentioned concerns about medication appropriateness or treatment burden. Cognitive impairment is one of the most challenging illnesses that primary care clinicians address because of the behavioral and psychological complications and because of structural barriers. ${ }^{43}$ This complexity, compounded by logistical obstacles (e.g., locating records from other clinicians or sites of care and reconciling medication lists), often left little time for discussions about optimal prescribing. For example, the transcripts revealed that medication reconciliation was time-consuming and complicated, particularly when companions were poorly equipped to clarify medication regimens; this is in line with a previous study that found that $96 \%$ of primary care provider medication lists contained discrepancies compared with what the patient was actually taking. ${ }^{44}$ In other cases, such as the example involving the companion of the 100-year-old patient with a MMSE score of 9, clinicians attempted to deprescribe (or to avoid starting a PIM) but the interactions went astray. Even if a clinician recognizes a medication as potentially inappropriate or a candidate for withdrawal, talking about deprescribing is difficult, especially in the context of cognitive impairment, with its prolonged and uncertain trajectory. It involves eliciting patients' goals and preferences, conveying complex benefit-harm information, and broaching the subject of illness trajectory without being perceived as giving up on the patient. ${ }^{18,35}$ Future efforts should focus on providing clinicians with frameworks or conversation guides to facilitate shared decision-making about medications. Such guides can be modeled after existing resources developed for advance care planning. ${ }^{45}$ Additional studies are needed to elicit patient and caregiver preferences regarding clinician communication about deprescribing and to examine how such communications are received and interpreted. These are important next steps before designing deprescribing conversation guides for clinicians as well as patient-directed educational materials. Patient-directed educational materials are essential to foster shared decision-making around medication use, and there is strong evidence that such materials work. ${ }^{46,}{ }^{47}$ In addition, further research is needed to understand how other health care providers, such as pharmacists and nurses, can contribute to deprescribing. ${ }^{48}$ Lastly, strategies are needed to help clinicians overcome complexity and technical or logistical hurdles during visits for patients with cognitive impairment and leave time for important discussions about medication appropriateness. ${ }^{49}$

This study has several limitations. Each of the examples we present reflects a single visit; a clinician may not have addressed deprescribing but may have done so during a previous interaction. We are only able to comment on how clinicians discussed deprescribing, not on situations in which they did not discuss it. The visits were recorded as part of the SAME Page trial, which may have affected whether medications were discussed but not how. One of the items on the SAME Page checklist was "managing or taking medications." Overall, $29 \%$ of patients and $33 \%$ of companions identified this as a visit priority. It is possible that inclusion of this item on the checklist encouraged patients and companions to discuss medications during the visits. It is also possible that there was a carryover effect for clinicians, such that they were more likely to discuss medications with patients and companions in both groups because exposure to patients in the intervention group altered their behavior. We also do not know how many of these conversations resulted in a drug being discontinued or not prescribed unless it was stated during the conversation. The study was conducted in the Baltimore metropolitan area and two of the clinics are affiliated with Johns Hopkins University (although the clinicians do not have academic appointments); therefore, the results may not generalize to other locations. The clinicians knew they were being recorded, which may have affected their behavior. Finally, we strived for objectivity but assessment of the elements of communication is inherently subjective. Certain quotes could be classified in multiple themes. Each transcript was independently read by 3 coders, with disagreements resolved through discussion.

\section{CONCLUSION}

Achieving optimal medication use for older adults with cognitive impairment is challenging. ${ }^{1,}{ }^{5-10}$ How best to foster informed decision-making about optimal medication use in older adults with cognitive impairment is unknown. Based on these transcripts, we identified many instances in which primary care clinicians incorporated deprescribing into routine clinical care. These examples can be used to help create deprescribing conversation guides for clinicians, as well as patient-directed educational materials. Yet, we also identified numerous challenges that prevented clinicians from working 
together with patients and companions to optimize prescribing. Future research should develop and test deprescribing interventions that seek to overcome these barriers for the growing population of older adults with cognitive impairment.

Corresponding Author: Ariel R. Green, MD, MPH, PhD; Division of Geriatric Medicine and Gerontology, Department of MedicineJohns Hopkins University School of Medicine, Baltimore, MD, USA (e-mail: ariel@jhmi.edu).

Funding Information Research reported in this publication was conducted with grant support from the National Institute on Aging under award numbers K23AG054742 (Green), 5R21AG049967-02 (Wolff), K24AG056578 (Boyd), and 5T35AG026758-14 (Chapman, Phung, Smith).

\section{Compliance with Ethical Standards:}

The study was approved by the institutional review board of the Johns Hopkins Bloomberg School of Public Health. The funding agency did not have a role in the design, conduct, or reporting of the study results.

Conflict of Interest: Dr. Boyd writes a chapter on multimorbidity for UpToDate, for which she receives a royalty. All remaining authors declare that they do not have a conflict of interest.

\section{REFERENCES}

1. Alzheimer's Association. 2015 Alzheimer's disease facts and figures. Alzheimers Dement. 2015;11(3):332-384.

2. Plassman BL, Langa KM, Fisher GG, et al. Prevalence of cognitive impairment without dementia in the United States. Ann Intern Med. 2008;148(6):427-434

3. Alzheimer's Association. 2018 Alzheimer's disease facts and figures. Alzheimers Dement. 2018;14(3):367-429.

4. Lyketsos CG. Neuropsychiatric Symptoms in Dementia: Overview and Measurement Challenges. J Prev Alzheimers Dis. 2015;2(3):155-156.

5. Elmstahl S, Stenberg I, Annerstedt $\mathbf{L}$, Ingvad B. Behavioral disturbances and pharmacological treatment of patients with dementia in family caregiving: a 2-year follow-up. Int Psychogeriatr. 1998;10(3):239252.

6. Lau DT, Mercaldo ND, Harris AT, Trittschuh E, Shega J, Weintraub S. Polypharmacy and potentially inappropriate medication use among community-dwelling elders with dementia. Alzheimer Dis Assoc Disord. 2010:24(1):56-63.

7. Johnell K. Inappropriate Drug Use in People with Cognitive Impairment and Dementia: A Systematic Review. Curr Clin Pharmacol. 2015;10(3): 178-184.

8. Castelino RL, Hilmer SN, Bajorek BV, Nishtala P, Chen TF. Drug Burden Index and potentially inappropriate medications in communitydwelling older people: the impact of Home Medicines Review. Drugs Aging. 2010;27(2):135-148.

9. Lin PJ, Fillit HM, Cohen JT, Neumann PJ. Potentially avoidable hospitalizations among Medicare beneficiaries with Alzheimer's disease and related disorders. Alzheimers Dement. 2013;9(1):30-38.

10. Patel A, Parikh R, Howell EH, Hsich E, Landers SH, Gorodeski EZ. Mini-cog performance: novel marker of post discharge risk among patients hospitalized for heart failure. Circ Heart Fail. 2015;8(1):8-16.

11. Carnahan RM, Lund BC, Perry PJ, Chrischilles EA. The concurrent use of anticholinergics and cholinesterase inhibitors: rare event or common practice? J Am Geriatr Soc. 2004;52(12):2082-2087.

12. Moga DC, Carnahan RM, Lund BC, et al. Risks and benefits of bladder antimuscarinics among elderly residents of Veterans Affairs Community Living Centers. J Am Med Dir Assoc. 2013;14(10):749-760.

13. Berdot S, Bertrand M, Dartigues JF, et al. Inappropriate medication use and risk of falls-a prospective study in a large community-dwelling elderly cohort. BMC Geriatr. 2009;9:30
14. Kay GG, Abou-Donia MB, Messer WS, Jr., Murphy DG, Tsao JW, Ouslander JG. Antimuscarinic drugs for overactive bladder and their potential effects on cognitive function in older patients. J Am Geriatr Soc. 2005;53(12):2195-2201.

15. Campbell N, Boustani M, Limbil T, et al. The cognitive impact of anticholinergics: a clinical review. Clin Interv Aging. 2009;4:225-233.

16. Sunderland T, Esposito G, Molchan SE, et al. Differential cholinergic regulation in Alzheimer's patients compared to controls following chronic blockade with scopolamine: a SPECT study. Psychopharmacology. 1995;121(2):231-241.

17. Gray SL, Anderson ML, Dublin S, et al. Cumulative use of strong anticholinergics and incident dementia: a prospective cohort study. JAMA Intern Med. 2015;175(3):401-407.

18. Reeve E, Bell JS, Hilmer SN. Barriers to Optimising Prescribing and Deprescribing in Older Adults with Dementia: A Narrative Review. Curr Clin Pharmacol. 2015;10(3):168-177.

19. Reeve E, Shakib S, Hendrix I, Roberts MS, Wiese MD. Review of deprescribing processes and development of an evidence-based, patientcentred deprescribing process. Br J Clin Pharmacol. 2014;78(4):738-747.

20. Scott IA, Hilmer SN, Reeve E, et al. Reducing inappropriate polypharmacy: the process of deprescribing. JAMA Intern Med. 2015;175(5):827-834.

21. Stacey D, Legare F, Col NF, et al. Decision aids for people facing health treatment or screening decisions. Cochrane Database Syst Rev. 2014;1:CD001431.

22. Karlawish J. Measuring Decision-Making Capacity in Cognitively Impaired Individuals. Neurosignals. 2008;16(1):91-98.

23. Vick JB, Amjad H, Smith KC, et al. "Let him speak": a descriptive qualitative study of the roles and behaviors of family companions in primary care visits among older adults with cognitive impairment. Int $J$ Geriatr Psychiatry. 2018;33(1):e103-e112.

24. Wolff JL, Roter DL, Boyd CM, et al. Patient-Family Agenda Setting for Primary Care Patients with Cognitive Impairment: the SAME Page Trial. J Gen Intern Med. 2018;33(9):1478-1486.

25. Callahan CM, Unverzagt FW, Hui SL, Perkins AJ, Hendrie HC. Sixitem screener to identify cognitive impairment among potential subjects for clinical research. Med Care. 2002;40(9):771-781.

26. Doing qualitative research. In: Crabtree BF, Miller WL, eds. Newbury Park: Sage Publications; 1992.

27. Boeije H. A Purposeful Approach to the Constant Comparative Method in the Analysis of Qualitative Interviews. Qual Quant. 2002;36(4):391-409.

28. Hsieh HF, Shannon SE. Three approaches to qualitative content analysis. Qual Health Res. 2005; 15(9):1277-1288.

29. Braddock CH, 3rd, Edwards KA, Hasenberg NM, Laidley TL, Levinson W. Informed decision making in outpatient practice: time to get back to basics. JAMA. 1999;282(24):2313-2320.

30. Elwyn G, Edwards A, Britten N. "Doing prescribing": how doctors can be more effective. BMJ. 2003;327(7419):864-867.

31. Mold JW, Hamm RM, McCarthy LH. The law of diminishing returns in clinical medicine: how much risk reduction is enough? J Am Board Fam Med. 2010;23(3):371-375

32. Farrell B, Black C, Thompson $\mathbf{W}$, et al. Deprescribing antihyperglycemic agents in older persons: Evidence-based clinical practice guideline. Can Fam Physician. 2017;63(11):832-843.

33. Bjerre LM, Farrell B, Hogel M, et al. Deprescribing antipsychotics for behavioural and psychological symptoms of dementia and insomnia. Can Fam Physician. 2018;64(1):17.

34. Reeve E, To J, Hendrix I, Shakib S, Roberts MS, Wiese MD. Patient barriers to and enablers of deprescribing: a systematic review. Drugs Aging 2013;30(10):793-807.

35. Anderson K, Stowasser D, Freeman C, Scott I. Prescriber barriers and enablers to minimising potentially inappropriate medications in adults: a systematic review and thematic synthesis. BMJ Open. 2014;4(12):e006544

36. Kerns JW, Winter JD, Winter KM, Kerns CC, Etz RS. Caregiver perspectives about using antipsychotics and other medications for symptoms of dementia. Gerontologist. 2018;58(2):e35-e45.

37. Reeve E, Low LF, Hilmer SN. Beliefs and attitudes of older adults and carers about deprescribing of medications: a qualitative focus group study. Br J Gen Pract. 2016;66(649):e552-560.

38. Reeve E, Wolff JL, Skehan M, Bayliss EA, Hilmer SN, Boyd CM. Assessment of attitudes toward deprescribing in older medicare beneficiaries in the United States. JAMA Intern Med. 2018;178(12):1673-1680.

39. Bynum JPW, Barre L, Reed C, Passow H. Participation of Very Old Adults in Healthcare Decisions. Med Decis Mak. .2014;34(2):216-230. 
40. Belcher VN, Fried TR, Agostini JV, Tinetti ME. Views of older adults on patient participation in medication-related decision making. J Gen Intern Med. 2006;21(4):298-303.

41. Pisters R, Lane DA, Nieuwlaat R, de Vos CB, Crijns HJ, Lip GY. A novel user-friendly score (HAS-BLED) to assess 1-year risk of major bleeding in patients with atrial fibrillation: the Euro Heart Survey. Chest. 2010; 138(5): 1093-1100

42. Lloyd-Jones DM, Braun LT, Ndumele CE, et al. Use of risk assessment tools to guide decision-making in the primary prevention of atherosclerotic cardiovascular disease. J Am Coll Cardiol. 2019;73(24):3153-3167.

43. Stewart TV, Loskutova N, Galliher JM, et al. Practice patterns, beliefs, and perceived barriers to care regarding dementia: a report from the American Academy of Family Physicians (AAFP) national research network. J Am Board Fam Med. 2014;27(2):275-283.

44. Frank C, Godwin M, Verma S, et al. What drugs are our frail elderly patients taking? Do drugs they take or fail to take put them at increased risk of interactions and inappropriate medication use? Can Fam Physician. 2001;47:1198-1204.

45. Sudore RL, Boscardin J, Feuz MA, McMahan RD, Katen MT, Barnes DE. Effect of the PREPARE Website vs an Easy-to-Read Advance Directive on Advance Care Planning Documentation and Engagement Among Veterans: A Randomized Clinical Trial. JAMA Intern Med.
2017;177(8):1102-1109.

46. Tannenbaum C, Martin P, Tamblyn R, Benedetti A, Ahmed S. Reduction of inappropriate benzodiazepine prescriptions among older adults through direct patient education: the EMPOWER cluster randomized trial. JAMA Intern Med. 2014; 174(6):890-898.

47. Martin P, Tamblyn R, Benedetti A, Ahmed S, Tannenbaum C. Effect of a Pharmacist-Led Educational Intervention on Inappropriate Medication Prescriptions in Older Adults: The D-PRESCRIBE Randomized Clinical Trial. JAMA. 2018;320(18):1889-1898.

48. Thompson W, Reeve E, Moriarty F, et al. Deprescribing: Future directions for research. Res Soc Adm Pharm. 2019;15(6):801-805.

49. Callahan CM, Sachs GA, Lamantia MA, Unroe KT, Arling G, Boustani MA. Redesigning systems of care for older adults with Alzheimer's disease. Health Aff (Millwood) 2014;33(4):626-632.

Publisher's Note: Springer Nature remains neutral with regard to jurisdictional claims in published maps and institutional affiliations.

Table 4 Characteristics of Patients and Companions Stratified by Perceived Quality of Medication Communication

\begin{tabular}{|c|c|c|c|c|}
\hline \multirow[t]{2}{*}{ Characteristic } & \multicolumn{2}{|c|}{$\begin{array}{l}\text { Perceived quality of } \\
\text { communication with clinician } \\
\text { about prescription medications* }\end{array}$} & \multirow[t]{2}{*}{$p$ Value } & \multirow[t]{2}{*}{ Total $(N=93)^{\dagger}$} \\
\hline & Low & High & & \\
\hline \multicolumn{5}{|l|}{ Patients } \\
\hline Age (years), mean (SD) & $81.1(7.0)$ & $77.7(6.9)$ & 0.03 & $79.9(7.6)$ \\
\hline Gender, no. $(\%)$ & & & 0.83 & \\
\hline Female & $20(48.8)$ & $21(51.2)$ & & $48(51.6)$ \\
\hline Male & $19(46.3)$ & $22(53.7)$ & & $45(48.4)$ \\
\hline Race/ethnicity, no. (\%) & & & 0.41 & \\
\hline White & $21(43.8)$ & $27(56.3)$ & & $54(58.1)$ \\
\hline Nonwhite, Hispanic, or other & $18(52.9)$ & $16(47.1)$ & & $39(41.9)$ \\
\hline Education, no. (\%) & & & 0.83 & \\
\hline High school or less & $20(48.8)$ & $21(51.2)$ & & $48(51.6)$ \\
\hline Beyond high school & $19(46.3)$ & $22(53.7)$ & & $45(48.4)$ \\
\hline Mini-Mental State Examination score, mean (SD) & $21.5(7.2)$ & $23.7(4.1)$ & 0.08 & $21.6(6.8)$ \\
\hline Number of prescription medications, no. (\%) & & & 0.15 & \\
\hline$<10$ & $28(47.5)$ & $31(52.5)$ & & $67(72.0)$ \\
\hline$>10$ & $8(40.0)$ & $12(60.0)$ & & $22(23.7)$ \\
\hline Not sure & $3(100.0)$ & $0(0.0)$ & & $4(4.3)$ \\
\hline \multicolumn{5}{|l|}{ Companions } \\
\hline Gender, no. $(\%)$ & & & 0.26 & \\
\hline Female & $28(40.6)$ & $41(59.4)$ & & $70(75.3)$ \\
\hline Male & $6(27.3)$ & $16(72.7)$ & & $23(24.7)$ \\
\hline Relationship to patient, no. (\%) & & & 0.31 & \\
\hline Spouse/partner & $15(42.9)$ & $20(57.1)$ & & $37(39.8)$ \\
\hline Adult child & $16(31.4)$ & $35(68.6)$ & & $51(54.8)$ \\
\hline Other (e.g., friend, other relative) & $3(60.0)$ & $2(40.0)$ & & $5(5.4)$ \\
\hline Education, no. (\%) & & & 0.89 & \\
\hline High school or less & $10(38.5)$ & $16(61.5)$ & & $26(28.0)$ \\
\hline Beyond high school & $24(36.9)$ & $41(63.1)$ & & $67(72.0)$ \\
\hline
\end{tabular}

*Asked as part of a 2-week follow-up questionnaire; low = poor, fair, good; high = excellent

tIn total, 93 patient-companion dyads participated in the study; 82 patients and 91 companions responded to the question about quality of medication communication 